\title{
Comment 2.2
}

\author{
RAGAN ROBERTSON
}

With the deluge of data generated daily by a knowledge transfer office (KTO), strategic decisions and operational functions rely on metrics to identify areas of improvement or focus. Given the complexity of tasks managed by a KTO, determining what metrics are measured is critical. Coming from an academic background, I've been taught to ask "what is the goal?" or "what is the question?" before setting forth and measuring something. Asking these questions first helps determine the variables being measured, as well as ensuring that the measurers and those who will review the metrics are all in agreement as to what is being asked and answered. When focusing on measuring the functions of a KTO, I ask questions such as "what can be measured?," "why measure?," and "how does a metric affect other metrics?" Addressing these questions typically results in identifying the core functions of a KTO and focusing on the counts of these actions, such as licenses executed and patent applications filed. The AUTM Licensing Activity Survey is an excellent source of some of these measurements, as are the annual reports produced by KTOs. Some of these metrics are also highlighted in Table 2.5 in this chapter. Accumulation of broadly applicable data in a centrally accessible database can help facilitate these goals. This also allows for KTOs to compare their metrics with those of their peers to establish benchmarks. Another benefit of creating data as a shareable resource is the ability to share data with other interested parties, including policymakers and academics. In this way, this practice can further benefit the knowledge transfer field as a whole in addition to the office itself.

As data become more available, and our economies become more complex, KTOs are being asked to do more and more functions. One reason is that the KTO acts as a node, a nexus where industry connects to 
research, where intellectual property connects to contracts, where business development connects to academic pursuits. These connections in and of themselves lead to additional metrics that can be measured. How many meetings with industry occurred in the last quarter? How many different patent application families were put under an exclusive license this past year? How many sponsored research agreements were executed with licensees? As KTOs function in more roles at the interface of academics and business, more measurable data are generated and can be analyzed. However, as the roles of our offices expand, we must ensure that the data we are generating and measuring can lead to potential actions and not just to the act of obtaining data. We must always ask why we are measuring something and what we can do to change it for the better.

Naturally, due to the nature of the knowledge managed by KTOs, offices are becoming more involved within the entrepreneurship ecosystem. Subdivisions within KTOs, and even entire new departments, are being formed to address this growing role. The function as a nexus causes KTOs to be well-positioned to have an incredible impact in this ecosystem. By having the connections to industry, as well as access to new research being conducted, KTOs can identify the opportunities where new research results can lead to the formation of a new company. The ultimate result is that KTOs can have a significant impact on the economy, especially at the local level. New companies formed, new jobs created, new revenue generated, and new taxes paid are some of the more easily identifiable benefits to economic output that can be sourced from the work performed by a KTO. This additional role for KTOs within the entrepreneurship ecosystem can also lead to unintended benefits, such as new institutional donors, or diversification of industry within a local ecosystem.

However, we do have to be careful to assign economic output to KTOs accurately and avoid generalization of data or misattribution of revenue. Skeptics of the industry have focused on return on investment (ROI), typically syncing research dollars sourced from a governmental agency and the licensing revenue received directly by a KTO. While this connection is an oversimplification, expanding to the other extreme of attributing all economic benefit generated by external entities connected to a KTO is also misleading. In addition to drawing conclusions based on overgeneralization of data, KTOs must also be wary of "paralysis of analysis" in which the goal is to gather more data without making a decision, or the confusion that results from an overabundance of data 
from multiple sources. This speaks directly to the question as to what the true impact is downstream of a KTO's activity. This harks back to asking ourselves the question of why we are measuring something and what we can do to change it for the better.

This chapter does an excellent job of identifying key metrics that should be measured by KTOs, along with the reasons why they are measured and the potential economic impact. While these metrics are clear and should be implemented by KTOs, the industry still lacks metrics to measure several important functions of KTOs. These include metrics to measure the effect of a KTO's work on societal impacts. As identified in this chapter, these impacts are very difficult to measure, typically relying on case studies such as those found in the AUTM Better World Project. Case studies, unfortunately, can be overly specific and their conclusions can be difficult to apply to general practice. However, there are metrics that could be used to address this impact. For example, for the past few years, AUTM has been collecting data regarding women inventors, specifically how many disclosures include a woman and how many new patent applications include a woman. What actions can or should be taken related to these data are only now being developed, but it is a start toward how to address a significant societal impact. Expanding on the theme of inclusion, metrics could also be collected related to race or ethnicity. KTOs may already have some of these data, as we must report citizenship within patent applications, but they have yet to be utilized. These data have the potential to have a far-reaching impact outside of the knowledge transfer or academic spheres, and it will be interesting to see what comes of them.

As identified, metrics are a key necessity for any KTO to inform strategic decisions and operational functions. Metrics need not only include the core functions, such as patent application filings and licenses executed, but also other important economic drivers, such as startups formed and investments raised. However, no matter what metrics a KTO measures, they are numbers for numbers' sake if the metrics are not aligned with a KTO's goals. Metrics must be actionable - how can a team affect them and what does that effect mean? By being clear and transparent as to how a metric informs and achieves its goals, a KTO is on the path to making an economic and societal impact. 